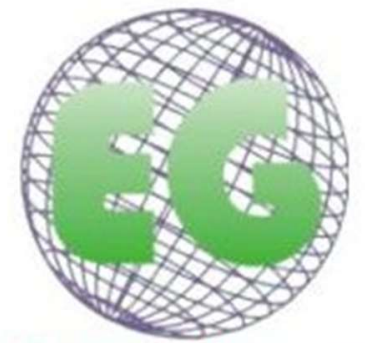

\title{
ORIGINALES
}

\section{Validez y consistencia interna del instrumento Vínculo entre padres e hijos neonatos}

Internal consistency and validity of the instrument Attachment between parents and newborn children

Alix Nathalya Vargas Vásquez ${ }^{1}$

Myriam Patricia Pardo Torres ${ }^{2}$

1 Magister en Enfermería Universidad Nacional de Colombia, Sede Bogotá. Docente Fundación Universitaria del Área Andina. Bogotá-Colombia. avargas83@areandina.edu.co

2 PhD en Ciencias de la Salud, ENSAP Habana-Cuba, Profesora asociada, Facultad de Enfermería, Universidad Nacional de Colombia, Sede Bogotá. Colombia.

\section{https://doi.org/10.6018/eglobal.403721}

Recibido: $19 / 11 / 2019$

Aceptado:18/01/2020

\section{RESUMEN:}

Objetivo: Determinar la validez y la consistencia interna del instrumento Vínculo entre padres e hijos neonatos versión en español resultado de la adaptación transcultural del Maternal Attachment Inventory (MAI) de Mary Müller.

Materiales y método: Estudio descriptivo metodológico - psicométrico, cuya validez facial y de contenido se logró con la participación de un panel de expertos y 385 potenciales padres con bajo nivel de escolaridad. Para la validez de constructo y consistencia interna la muestra fue de 352 padres de recién nacidos a término.

Resultados: La validez facial para la población general mostró una comprensión global y por ítems superior a $90 \%$, y un índice de aceptabilidad superior en las tres categorías valoradas; igualmente el índice de Kapa y Fleiss mostró concordancia interjueces para cada uno de los ítems por encima de 0.69. La validez de contenido global presenta fuerte pertinencia y relevancia con un índice de Kapa y Fleiss de 0.71 y 0.77 respectivamente. En la validez de constructo el análisis factorial exploratorio arrojó cuatro factores con una varianza total explicada por el índice de Kaiser-Meyer-Olkin (KMO) de 73 $\%$, y la consistencia interna por el Alfa de Cronbach fue de 0.7 para el total de la escala.

Conclusiones: El instrumento Vínculo entre padres e hijos neonatos es válido y confiable para medir el vínculo afectivo en el contexto colombiano cuyo uso favorece la práctica de enfermería basada en evidencia en contextos diversos como Unidades de Cuidado Neonatal y en la práctica ambulatoria.

Palabras clave: Vínculo afectivo, Apego, Validez, Confiabilidad, Psicometría.

\section{ABSTRACT:}

Objective: To determine the validity and internal consistency of the instrument Attachment between parents and newborn children, spanish version as a result from the transcultural adaptation of the Maternal Attachment Inventory (MAI) by Mary Muller.

Materials and method: Methodological - psychometric descriptive study, whose facial and content validity was achieved with the participation of a panel of experts and 385 potential parents with low level 
of schooling. For the validity of construct and internal consistency the sample was 352 parents of fullterm newborns.

Results: Facial validity for the general population showed a global and item understanding of more than $90 \%$, and a higher acceptability rate in the three categories valued; likewise, the Kapa and Fleiss index showed inter-concordance for each of the items above 0.69 . The validity of global content has a strong pertinence and relevance with a Kapa and Fleiss index of 0.71 and 0.77 respectively. In the validity of the construct, the exploratory factor analysis yielded four factors with a total variance explained by the Kaiser-Meyer-Olkin Index (KMO) of $73 \%$, and the internal consistency by Cronbach's Alpha was 0.7 for the total of the scale.

Conclusions: The Attachment between parents and newborn children instrument is valid and reliable to measure the affective attachment in the Colombian context whose use favors the practice of evidencebased nursing in diverse contexts such as Neonatal Care Units and in ambulatory practice.

Keywords: Affective attachment, validity, reliability, psychometry.

\section{INTRODUCCIÓN}

El vínculo afectivo abordado desde las ciencias humanas, principalmente, se inicia con los estudios de John Bowlby en 1951 relacionados con el cuidado maternal y la salud mental de los cuales se deriva la teoría del apego (1). En la década de los cincuenta se asocia Ainsworth con Bowlby para trabajar en el desarrollo de la teoría, trabajo del que surge la clasificación de los tipos de apego. (2). Y como aporte a esta línea de conocimiento los pediatras Kennell y Klaus en la década de los 80 avanzan en el conocimiento del Bonding que hace referencia al vínculo del hijo con su madre en las primeras horas posteriores al nacimiento. ${ }^{(3)}$. Por otra parte, la enfermera Reva Rubín en su práctica profesional inicia el estudio de la observación del acercamiento madre hijo en el periodo neonatal y su importancia para el establecimiento vincular y la consolidación del rol materno.

A partir de los avances en la temática alcanzados por Rubín y Bowlby la teórica Ramona Mercer fortalece el fundamento para el desarrollo de la teoría de Convertirse en madre que plantea la conexión entre la posibilidad de llevar a cabo exitosamente el proceso de ejercer la maternidad y el apego. (4). La consecución del rol y el fortalecimiento del vínculo madre hijo es evidente en los conceptos propios de la teoría de Mercer, en los que se describe la unión/interacción como un asunto que facilita el origen del vínculo afectivo y emocional entre dos individuos y hace parte del rol parental, aunado al apoyo emocional, y al apoyo informativo que ofrecen herramientas para afrontar las situaciones de estrés en el ejercicio de la maternidad(4).

Para valorar el vínculo afectivo es necesario disponer de indicadores empíricos válidos y confiables que faciliten su medición, sin embargo, existe controversia respecto a la forma más conveniente de hacerlo, bien sea a través de la observación directa o de la expresión de emociones y sentimientos ${ }^{(5)}$.

Con base en la importancia que tiene la promoción y fortalecimiento del vínculo afectivo en el desarrollo personal y teniendo en cuenta que Enfermería como disciplina y profesión tiene un rol protagónico en la valoración y consolidación del vínculo afectivo entre padres e hijo, al proveer un cuidado materno perinatal temprano, se llevó a cabo una pesquisa con el objeto de tener a la mano un indicador empírico valido y confiable para valorar el vínculo afectivo entre padres o madres y sus hijos neonatos nacidos a término, con la opción de utilizarlo en el ámbito clínico y en el contexto propio del hogar, de esta tarea se seleccionó el Maternal Attachment 
Inventory (MAI) de Mary Müller instrumento que se caracteriza por tener parámetros psicométricos válidos y que fue realizado por una enfermera para diadas de neonatos hospitalizados en Unidades de Cuidado Neonatal y en el escenario ambulatorio. ${ }^{(6)}$.

Partiendo de la premisa de que enfermería en el contexto colombiano requiere identificar en la cotidianidad los comportamientos de los padres con sus hijos recién nacidos a término relacionados con el vínculo afectivo, y planificar a partir de estas observaciones un cuidado acorde a la realidad con la intención de abordar el núcleo familiar del neonato, se procedió a realizar en una primera fase la adaptación transcultural del Maternal Attachment Inventory ya que sólo se disponía de la versión en idioma ingles de Müller ${ }^{(6)}$ y de una versión en taiwanés de Yu, Hung, Chan, Yeh, and Lai (7). Como resultado es este procedimiento surge el instrumento que se denominó Vínculo entre padres e hijos neonatos para ser diligenciado por madres y padres, con 24 ítems agrupados en cuatro dimensiones coherentes con los conceptos de la teoría Convertirse en madre de Ramona Mercer (8), del cual este artículo da cuenta del proceso de validez y confiabilidad.

\section{OBJETIVO}

Determinar la validez y la consistencia interna del instrumento Vínculo entre padres e hijos neonatos versión en español resultado de la adaptación transcultural del Maternal Attachment Inventory (MAI) de Mary Müller.

\section{MATERIAL Y MÉTODOS}

Estudio descriptivo metodológico de carácter psicométrico ${ }^{(9)}$, avalado por el Comité de Ética de la Facultad de Enfermería de la Universidad Nacional de Colombia Sede Bogotá (Acto Administrativo Acta 8 de 25 de mayo de 2015. Aval-012-2015).

\section{Muestra}

La validez facial del instrumento se realizó a partir de una muestra de 385 potenciales padres en edad reproductiva, alfabetos, con bajo nivel de escolaridad (atendiendo al principio de parsimonia de Navaja de Ockham (10). Para estimar estadísticamente la comprensibilidad de los 24 ítems del instrumento se asumió una confianza del $95 \%$ y un error máximo admisible del $5 \%$. Para la validez facial y de contenido se seleccionó un grupo de cinco profesionales de la salud especialistas en el área materna perinatal, con experiencia en investigación y arbitraje de estudios metodológicos. La validez de constructo y la consistencia interna se obtuvieron a partir de una muestra de 352 padres, determinada a partir de la teoría clásica de validez de un instrumento de Streiner y Norman (11), que sugiere para efectos del análisis factorial un rango entre 5 a 20 individuos por ítem, en este caso el número de participantes fue de quince por cada ítem. Los participantes debían ser madres y padres de neonatos nacidos a término, cuya selección se realizó mediante un muestreo aleatorio simple ${ }^{(12) .}$

\section{Procedimiento}

El instrumento Vínculo entre padres e hijos neonatos surge del ejercicio investigativo de adaptación transcultural del idioma inglés al español de la escala Maternal Attachment Inventory -MAl- de Mary Mûller ${ }^{(6)}$ cuyas bases teóricas derivan de las ciencias humanas con los aportes de Bowlby y desde enfermería a partir de los 
conceptos de Rubín. Mercer y Ferketich. El procedimiento de adaptación transcultural del Maternal Attachment Inventory (MAI) se desarrolló en dos etapas, la primera comprendió la selección del instrumento y la segunda la adaptación transcultural propiamente dicha, que como lo indica Tristán-López ${ }^{(13)}$ se realiza a partir de tres fases como se muestra más adelante en el texto.

\section{Primera etapa. Selección del instrumento}

Se escogió el instrumento Maternal Attachment Inventory (MAI) de Mary Müller porque además de ser un indicador empírico válido y confiable, su autora lo diseño para ser aplicado en escenarios clínicos y ambulatorios, para valorar el vínculo afectivo entre padres o madres y sus hijos neonatos nacidos a término. ${ }^{(6)}$

El instrumento MAl en su versión original está compuesto por 26 ítems, con una escala Tipo Likert que arroja un puntaje mínimo de 26 y un máximo de 104 cuya interpretación corresponde a mayor puntuación mayor apego afectivo. El instrumento expresa validez facial, de contenido, de constructo y fiabilidad, su autora realizó la validez convergente y la concurrente con tres instrumentos que miden apego y adaptación materna, ellos son el HIFBN, el MSAS y el PPMAMA, el resultado de este proceso mostró que dos instrumentos reportaron adecuados niveles de correlación MSAS $(0,71)$ y PPMAMA $(0,64)$ con una significancia $<0.01$, por tanto se reporta una validez convergente adecuada, ya que los valores de correlación del MAI con los "Gold standar" indicaron que mide el mismo fenómeno. El alfa de Cronbach dio como resultado $0,85^{(6)}$

\section{Segunda etapa. Adaptación transcultural}

A partir de las recomendaciones de Tristán ${ }^{(13)}$ se procedió a realizar el procedimiento de adaptación transcultural en tres fases (ver figura 1)

Figura 1. Adaptación transcultural del Maternal Attachment Inventory (MAI)

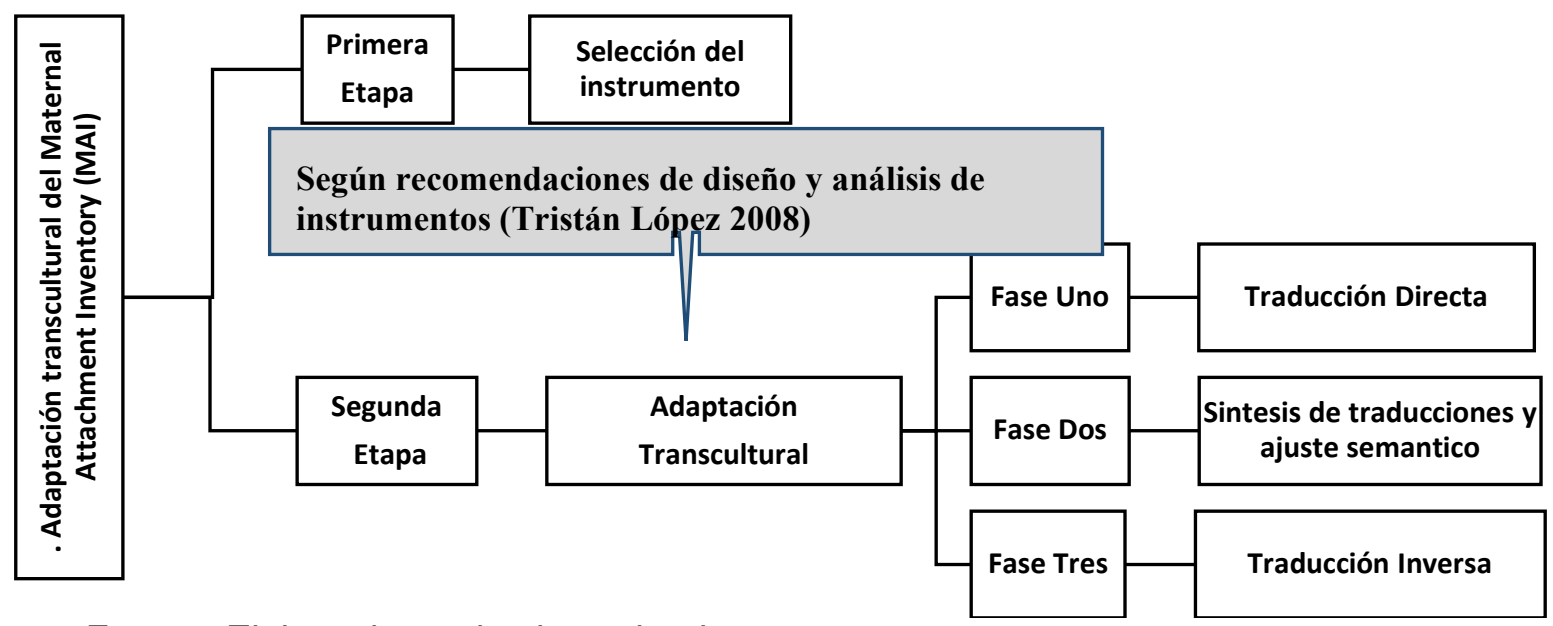

Fuente: Elaborado por las investigadoras

\section{Fase 1. Traducción directa}

Procedimiento en el que participaron tres traductores oficiales certificados, uno de ellos correspondía a una enfermera bilingüe de nacionalidad colombiana y con experticia en el área materno perinatal que se desempeña como profesional de la 
salud en Estados Unidos. Los dos intérpretes realizaron la traducción simultáneamente y ofrecieron la versión más cercana a la escala original del MAI en cuanto a gramática y contexto. ${ }^{(10)}$.

\section{Fase 2. Síntesis de traducciones y Ajuste semántico}

En esta fase participó un grupo conformado por un lingüísta, un estadístico, el director de tesis y el investigador principal, con el propósito de indagar la redacción, el fraseo y el contenido de cada uno de los 26 ítems del instrumento. Con el resultado de las traducciones se realizaron adecuaciones y cambios según el contexto colombiano, se valoró la redacción, el sentido de las oraciones, y la coherencia del contenido con el apoyo del lingüista quien cabe anotar estaba familiarizado con los términos médicos, sintetizando las traducciones en una versión preliminar.

Este procedimiento arrojó similitudes de la versión original del MAI, entre los ítems 11, 10 y 20 con los ítems 13, 16 y 23 respectivamente, razón que llevo al grupo de expertos a suprimir estos últimos tres ítems de la traducción. Por otra parte, el ítem 2 de la versión original del MAl en la traducción dio lugar a dos ítems diferentes. Como resultado la síntesis de las traducciones arroja una nueva versión compuesta por 24 ítems.

\section{Fase tres. Traducción inversa.}

Con la participación de los tres traductores oficiales se realizó la retro traducción al inglés de la versión del instrumento en español resultante de la fase dos. El producto de este proceso fue enviado a Mary Müller, quien en su concepto dio vía libre para continuar con el proceso de validez y confiabilidad del nuevo instrumento, que para ella presentaba diferencias respecto al original.

El nuevo instrumento denominado Vínculo entre padres e hijos neonatos fue sometido a pruebas psicométricas de validez facial, de contenido, de constructo y consistencia interna. ${ }^{(8)}$.

\section{Validez facial y de contenido del instrumento Vínculo entre padres e hijos neonatos}

La determinación estadística de la validez facial y de contenido se realizó con el Índice de Lawshe modificado y Kappa y Fleiss. El índice de Lawshe modificado determina el número de acuerdos entre expertos, este método valora la opinión del panel de expertos de los ítems del instrumento, y califica el instrumento en general y por ítems. (13). Y el índice de Kappa de Fleiss comprueba la concordancia entre los observadores para determinar el grado de aceptación y de similitud entre las respuestas dadas a la valoración de cada ítem por los jueces. (14)

Para esta investigación, se consideró como resultados satisfactorios en los índices de Lawshe modificado y Kappa y Fleiss aquellos ítems que obtuvieran un grado de aceptabilidad superior con valores entre 0,61 y 0,80 que demuestran un acuerdo sustancial aceptable, y un valor igual o superior a 0,81 .

Con la participación del grupo de potenciales padres y el panel de expertos, la validez facial se centró en valorar la comprensión, claridad y precisión. Concebidas, la comprensión como el entendimiento que existe al leer un ítem; la claridad como el tipo 
de lenguaje empleado y la manera de redactarlo y la precisión como el uso de un lenguaje conciso y exacto para evitar ambigüedades.

La validez de contenido valorada por el panel de expertos se orientó a resolver los criterios de pertinencia y relevancia de los 24 ítems que conforman el instrumento, con opciones de respuesta presentadas como relevante, poco relevante y no relevante, pertinente, poco pertinente y no pertinente; con el fin de determinar si los reactivos del instrumento representan de manera adecuada el universo del contenido.

$Y$ el grupo de potenciales padres realizaron la verificación del fraseo del instrumento en su totalidad, determinando la comprensión, claridad y precisión.

La validez de constructo y la consistencia interna se logró con un grupo de participantes resultantes de un muestreo intencional con el fin de garantizar la representatividad frente al fenómeno a evaluar por el instrumento. La estimación estadística del constructo se hizo a través del análisis factorial exploratorio de factores comunes cuyo propósito es establecer las estructuras internas y cruzadas de un conjunto de variables, y proveer un estimado de la carga factorial del instrumento en cada uno de las partes y en su totalidad (15).

La determinación de la consistencia interna del instrumento se hizo con el Alfa de Cronbach, se midió la sensibilidad al cambio, y el grado de consistencia interna de la totalidad y cada una de las dimensiones que componen el instrumento. (16). El Alfa de Cronbach según George y Mallery por debajo de 0,5 muestra un nivel no aceptable de fiabilidad, un valor de 0.70 a 0.90 se acepta como medida de homogeneidad de un instrumento.

\section{RESULTADOS}

El instrumento Vínculo entre padres e hijos neonatos contiene 24 ítems con escala de medición tipo Likert de cinco opciones, que puntúan entre 0 y 4 . La escala esta dividida en dos grupos, que están determinados por los criterios de intensidad y frecuencia, los primeros doce ítems valoran la intensidad con una escala que contiene los criterios Muy intensamente, Intensamente, Moderadamente, Levemente, Definitivamente no, y los doce ítems restantes valoran la frecuencia con una escala que contiene los parámetros Siempre, Casi siempre, Algunas veces, Pocas veces, y Nunca. (Ver tabla 1)

Tabla 01. Instrumento: Vínculo entre padres e hijos neonatos.

\begin{tabular}{|l|l|l|l|l|l|}
\hline Afirmación & $\begin{array}{l}\text { Muy } \\
\text { intensa } \\
\text { mente }\end{array}$ & $\begin{array}{l}\text { Intensa } \\
\text { mente }\end{array}$ & $\begin{array}{l}\text { Moderada } \\
\text { mente }\end{array}$ & $\begin{array}{l}\text { Leveme } \\
\text { nte }\end{array}$ & $\begin{array}{l}\text { Definiti- } \\
\text { vamente } \\
\text { no }\end{array}$ \\
\hline 1. Siento amor por mi bebé & & & & & \\
\hline 2. Siento ternura por mi bebé & & & & & \\
\hline 3. Siento felicidad con mi bebé & & & & & \\
\hline $\begin{array}{l}\text { 4. Hago planes futuros con mi } \\
\text { bebé }\end{array}$ & & & & & \\
\hline $\begin{array}{l}\text { 5. Sé que mi bebé necesita de } \\
\text { mi }\end{array}$ & & & & & \\
\hline $\begin{array}{l}\text { 6. Siento felicidad cuando mi } \\
\text { bebé esta alegre }\end{array}$ & & & & & \\
\hline
\end{tabular}




\begin{tabular}{|c|c|c|c|c|c|}
\hline $\begin{array}{l}\text { 7. Siento que mi bebé está } \\
\text { seguro conmigo }\end{array}$ & & & & & \\
\hline $\begin{array}{l}\text { 8. Siento que mi bebé es } \\
\text { bonito }\end{array}$ & & & & & \\
\hline $\begin{array}{l}\text { 9. Estoy contento (a) de que } \\
\text { este bebé sea mío }\end{array}$ & & & & & \\
\hline $\begin{array}{l}\text { 10. Siento felicidad cuando mi } \\
\text { bebé hace cosas nuevas }\end{array}$ & & & & & \\
\hline $\begin{array}{l}\text { 11. Disfruto tener a mi bebé } \\
\text { cerca de mi }\end{array}$ & & & & & \\
\hline $\begin{array}{l}\text { 12. Siento que soy importante } \\
\text { para mi bebé }\end{array}$ & & & & & \\
\hline Afirmación & Siempre & $\begin{array}{l}\text { Casi } \\
\text { Siempre }\end{array}$ & $\begin{array}{l}\text { Algunas } \\
\text { Veces }\end{array}$ & $\begin{array}{l}\text { Pocas } \\
\text { Veces }\end{array}$ & Nunca \\
\hline $\begin{array}{l}\text { 13. El tiempo que paso con } \mathrm{mi} \\
\text { bebé es especial }\end{array}$ & & & & & \\
\hline $\begin{array}{l}\text { 14. Me gusta tener contacto con } \\
\text { mi bebé }\end{array}$ & & & & & \\
\hline $\begin{array}{l}\text { 15. Les hablo a los demás sobre } \\
\text { mi bebé }\end{array}$ & & & & & \\
\hline 16. Disfruto estar con mi bebé & & & & & \\
\hline 17. Pienso en mi bebé & & & & & \\
\hline $\begin{array}{l}\text { 18. Reconozco las expresiones } \\
\text { y gestos de mi bebé }\end{array}$ & & & & & \\
\hline $\begin{array}{l}\text { 19. Es fácil expresarle amor a } \\
\text { mi bebé }\end{array}$ & & & & & \\
\hline $\begin{array}{l}\text { 20. Me da gusto sentir a mi } \\
\text { bebé }\end{array}$ & & & & & \\
\hline $\begin{array}{l}\text { 21. Estoy orgulloso (a) de } \mathrm{mi} \\
\text { bebé }\end{array}$ & & & & & \\
\hline $\begin{array}{l}\text { 22. Estoy pendiente de los } \\
\text { cuidados de mi bebé }\end{array}$ & & & & & \\
\hline $\begin{array}{l}\text { 23. Estoy pendiente de mi bebé } \\
\text { mientras duerme }\end{array}$ & & & & & \\
\hline $\begin{array}{l}\text { 24. Consuelo a mi bebé cuando } \\
\text { llora }\end{array}$ & & & & & \\
\hline
\end{tabular}

Fuente: Vargas-Vásquez, A. (2016). Validación del Instrumento "Vínculo Entre Padres E Hijos Neonatos" a partir del Maternal Attachment Inventory. Tesis requisito para optar al título de: Magíster en Enfermería Universidad Nacional de Colombia. Pág.122. Disponible en: http://bdigital.unal.edu.co/52842/1/52713456.2016.pdf

Para la interpretación de los resultados del instrumento se utilizó el criterio de Dalenius-Hodgers ${ }^{(17)}$, con el que se determina que un puntaje total menor o igual a 85 puntos indica un Vínculo afectivo entre padres e hijos neonatos bajo, un puntaje entre 86 y 89 puntos indica un vínculo afectivo entre padres e hijos neonatos medio o moderado y cuando el puntaje total es mayor a 90 puntos el vínculo afectivo entre padres e hijos neonatos es alto.

\section{Validez facial del instrumento Vínculo entre padres e hijos neonatos}

Los participantes que conformaron la muestra de la validez facial eran el $26 \%$ hombres (102) y el $74 \%$ mujeres (286). Con relación al nivel educativo, el $25 \%$ tenían un nivel educativo inferior a primaria, el $34 \%$ primaria completa, el $7 \%$ nivel medio incompleto, el $19 \%$ nivel medio completo, y el $15 \%$ con nivel secundaria. 
El nivel de comprensibilidad global del instrumento Vínculo entre padres e hijos neonatos fue del $98 \%$ y el análisis de comprensión por ítem el resultado estuvo por encima del $97 \%$, es decir, los participantes calificaron el instrumento en cuanto a su comprensión en un nivel superior. (Ver tabla 02).

Tabla 02: Validez Facial por población general del instrumento Vínculo entre padres e hijos neonatos.

\begin{tabular}{|l|l|}
\hline Ítem & $\begin{array}{l}\% \\
\text { Comprensibilidad } \\
\text { Neta }\end{array}$ \\
\hline 1. Siento amor por mi bebé & 98 \\
\hline 2. Siento ternura por mi bebé & 99 \\
\hline 3. Siento felicidad con mi bebé & 97 \\
\hline 4. Hago planes futuros con mi bebé & 98 \\
\hline 5. Sé que mi bebé necesita de mi & 98 \\
\hline 6. Siento felicidad cuando mi bebé esta alegre & 98 \\
\hline 7. Siento que mi bebé está seguro conmigo & 98 \\
\hline 8. Siento que mi bebé es bonito & 98 \\
\hline 9. Estoy contento (a) de que este bebé sea mío & 98 \\
\hline 10. Siento felicidad cuando mi bebé hace cosas nuevas & 99 \\
\hline 11. Disfruto tener a mi bebé cerca de mi & 97 \\
\hline 12. Siento que soy importante para mi bebé & 98 \\
\hline 13. El tiempo que paso con mi bebé es especial & 98 \\
\hline 14. Me gusta tener contacto con mi bebé & 98 \\
\hline 15. Les hablo a los demás sobre mi bebé & 98 \\
\hline 16. Disfruto estar con mi bebé & 98 \\
\hline 17. Pienso en mi bebé & 99 \\
\hline 18. Reconozco las expresiones y gestos de mi bebé & 98 \\
\hline 19. Es fácil expresarle amor a mi bebé & 99 \\
\hline 20. Me da gusto sentir a mi bebé & 99 \\
\hline 21. Estoy orgulloso (a) de mi bebé & 99 \\
\hline 22. Estoy pendiente de los cuidados de mi bebé & 98 \\
\hline 23. Estoy pendiente de mi bebé mientras duerme & 99 \\
\hline 24. Consuelo a mi bebé cuando llora & 97 \\
\hline
\end{tabular}

Fuente. Vargas-Vásquez, A. (2016). Validación del Instrumento "Vínculo Entre Padres E Hijos Neonatos" a partir del Maternal Attachment Inventory. Tesis requisito para optar al título de: Magíster en Enfermería Universidad Nacional de Colombia. Pág.122. Disponible en: http://bdigital.unal.edu.co/52842/1/52713456.2016.pdf

Por otra parte, la tabla 03 da cuenta de los resultados de los índices de Lawshe modificado y Kappa de Fleiss para los criterios de Claridad, Comprensión y Precisión. En el índice de Lawshe modificado el resultado del valor de cada uno de los criterios fue superior al mínimo preestablecido $(0,528)$, y con respecto al índice de Kappa de Fleiss en el análisis de concordancia inter observadores, se verifico que el resultado estuvo por encima de 0,69 en cada uno de los criterios con lo que se demuestra un fuerte grado de concordancia, resultados que llevan a inferir que la validez facial del instrumento es satisfactoria para ser aplicado al contexto colombiano. 
Tabla 03. Validez facial por expertos: Índices de Lawshe y Kappa de Fleiss del instrumento Vínculo entre padres e hijos neonatos.

\begin{tabular}{|l|l|l|}
\hline Criterio & $\begin{array}{l}\text { Índice de Lawshe } \\
\text { modificado }\end{array}$ & Índice de Kappa de Fleiss \\
\hline Claridad & 0,69 & 0,69 \\
\hline Comprensión & 0,75 & 0,75 \\
\hline Precisión & 0,73 & 0,73 \\
\hline
\end{tabular}

Fuente. Vargas-Vásquez, A. (2016). Validación del Instrumento "Vínculo Entre Padres E Hijos Neonatos" a partir del Maternal Attachment Inventory. Tesis requisito para optar al título de: Magíster en Enfermería Universidad Nacional de Colombia. Pág.125. Disponible en: http://bdigital.unal.edu.co/52842/1/52713456.2016.pdf

\section{Validez de Contenido del instrumento Vínculo entre padres e hijos neonatos}

Para esta fase del proceso de validez del instrumento participaron los expertos evaluadores de la fase de validez facial (ver tabla 04), con el propósito de determinar la pertinencia y la relevancia de los ítems del instrumento, él envio del reactivo a los expertos se hizo vía electrónica con las debidas orientaciones y una escala Likert con opción de respuesta presentadas como relevante, poco relevante y no relevante, pertinente, poco pertinente y no pertinente para cada uno de los ítems, con espacio para observaciones.

\section{Tabla 04. Características de expertos participantes en la validez facial y de contenido.}

Experto 1. Enfermera. Magister en Enfermería con Énfasis en Materno Perinatal. Enfermera de Cuidado Intensivo Neonatal del Hospital Militar Central. Docente Ocasional Especialización en Enfermería Materno Perinatal de la Universidad Nacional De Colombia. Investigadora en el área.

Experto 2. Enfermera, Magister en Enfermería con Énfasis en Materno Perinatal. Candidata a Doctora en Enfermería Facultad de Enfermería Universidad Nacional de Colombia. Investigadora en el área y experta en validación de instrumentos.

Experto 3. Enfermera, Magister en Enfermería con Énfasis en Materno Perinatal. Profesor Asociado Universidad Nacional de Colombia, Candidata a Doctora en Enfermería Facultad de Enfermería Universidad Nacional de Colombia. Investigadora en el área y experta en validación de instrumentos.

Experta 4. Enfermera, Especialista en Cuidado Intensivo Pediátrico. Magister en enfermería con Énfasis en Materno Perinatal, Candidata a Doctora en Enfermería Universidad Nacional de Colombia. Docente Universidad del Tolima.

Experta 5. Enfermera, Especialista en Gerencia de Servicios de Salud. Enfermera sala de partos de la Clínica de Marly. Docente Fundación Universitaria del Área Andina.

Fuente. Vargas-Vásquez, A. (2016). Validación del Instrumento "Vínculo Entre Padres E Hijos Neonatos" a partir del Maternal Attachment Inventory. Tesis requisito para optar al título de: Magíster en Enfermería Universidad Nacional de Colombia. Págs. 126 - 127. Disponible en: http://bdigital.unal.edu.co/52842/1/52713456.2016.pdf.

La tabla 05 muestra el resultado de la validez de contenido que arrojó el resultado del análisis del panel de expertos en los criterios de relevancia y pertinencia cuyos valores son satisfactorios porque demuestran un alto grado de acuerdo en el índice de Lawshe Modificado con valores por encima de 0.5823 , y con el índice de Kappa de Fleiss que mostró por su parte un grado de acuerdo fuerte con valores de relevancia y pertinencia por encima de 0,61 . 
Tabla 05. Validez de contenido global por expertos: Índices de Lawshe y Kappa de Fleiss del instrumento Vínculo entre padres e hijos neonatos.

\begin{tabular}{|l|l|}
\hline Índice de Lawshe Modificado & Índice de Kappa de Fleiss \\
\hline Relevancia: 0,93 & Relevancia: 0,71 \\
\hline Pertinencia: 0,92 & Pertinencia: 0,77 \\
\hline
\end{tabular}

Fuente. Vargas-Vásquez, A. (2016). Validación del Instrumento "Vínculo Entre Padres E Hijos Neonatos" a partir del Maternal Attachment Inventory. Tesis requisito para optar al título de: Magíster en Enfermería Universidad Nacional de Colombia. Pág.130. Disponible en: http://bdigital.unal.edu.co/52842/1/52713456.2016.pdf.

\section{Validez de Constructo del Instrumento Vínculo Entre Padres E Hijos Neonatos}

En esta fase del proceso de validación del instrumento se convocó un grupo de padres de neonatos nacidos a término con el fin de demostrar si la estructura teórica se conservaba en la nueva versión adaptada del MAl; para ello se tuvo en cuenta la teoría clásica de validez de instrumentos que según Streiner y Norman para efectos del análisis factorial se asume una muestra de 5 a 20 individuos por ítem, lo cual llevó a que se tuviese una muestra por el número de ítems entre 120 y 480 participantes.

Finalmente, la muestra para la validez de constructo estuvo constituida por 352 padres de recién nacidos a término $29,5 \%$ hombres (104) y $70,5 \%$ mujeres (248), $39 \%$ tenían estudios de primaria, $55 \%$ en nivel intermedio y $27 \%$ con nivel secundaria. La edad de los participantes osciló entre 18 y 50 años, y el $52,7 \%$ se ubicaba en el rango de 18 a 30 años.

Los resultados de la validez de constructo confirman que el fenómeno a medir es el vínculo afectivo entre padres e hijos neonatos, el test de Kaiser-Meyer-Olkin (KMO) fue de 0,732 valor que entre más se acerque a la unidad muestra una importante asociación entre las variables. Este valor indica que el instrumento Vínculo afectivo entre padres e hijos neonatos explica el $73 \%$ de la varianza, es decir mide un $73 \%$ del vínculo afectivo fenómeno para el cual fue diseñado. ${ }^{(18)}$.

En el análisis de la estructura del constructo Vínculo afectivo entre padres e hijos neonatos se aplicó el método de análisis factorial exploratorio, con el cual se extrajeron cada uno de sus factores a través del método de componentes principales y para la interpretación se utilizó la rotación Varimax, procedimiento que dio origen a cuatro factores, como lo muestra la tabla 06.

Tabla 06. Resultado de la agrupación de los ítems del instrumento Vínculo entre padres e hijos neonatos.

\begin{tabular}{|l|l|}
\hline Factor & Ítem \\
\hline 1 & $7,14,15,16,19$ y 24 \\
\hline 2 & $1,2,3,4,5,6$ y 20 \\
\hline 3 & $10,11,12,13,21$ y 23 \\
\hline 4 & $8,9,17,18$ y 22 \\
\hline
\end{tabular}

Fuente. Vargas-Vásquez, A. (2016). Validación del Instrumento "Vínculo Entre Padres E Hijos Neonatos" a partir del Maternal Attachment Inventory. Tesis requisito para optar al título de: Magíster en Enfermería Universidad Nacional de Colombia. Pág.136. Disponible en: http://bdigital.unal.edu.co/52842/1/52713456.2016.pdf 


\section{Interpretación de factores resultantes de la rotación Varimax}

Teniendo en cuenta que las bases teóricas del instrumento hacen parte de la teoría Convertirse en madre, de Ramona Mercer, se analizó la coherencia de los factores encontrados en la rotación Varimax con los conceptos de la teoría, en este ejercicio se encontró concordancia entre los conceptos apoyo emocional, unión - interacción, apoyo informativo y estrés y los ítems agrupados por factor. (Ver tabla 07).

Tabla 07. Relación entre conceptos teóricos y la agrupación por factores Factor uno: Concepto Apoyo emocional

\begin{tabular}{|l|l|}
\hline Definición del concepto & $\begin{array}{l}\text { Ítems agrupados que corresponden al } \\
\text { concepto }\end{array}$ \\
\hline & $\begin{array}{l}\text { 7: Siento que mi bebé está seguro conmigo. } \\
\text { 14: me gusta tener contacto con mi bebé. }\end{array}$ \\
$\begin{array}{l}\text { Sentirse amado, cuidado, digno } \\
\text { de confianza y comprendido. les hablo a los demás sobre mi bebé. } \\
\text { (Mercer, 1986). }\end{array}$ & $\begin{array}{l}\text { 16: disfruto estar con mi bebé. } \\
\text { 19: es fácil expresarle amor a mi bebé. } \\
\text { 24: consuelo a mi bebé cuando llora. }\end{array}$ \\
\hline
\end{tabular}

Factor dos: Concepto Unión - Interacción

Proceso por el cual se crea un 1. El tiempo que paso con mi bebé es especial. vínculo afectivo y emocional con 2. Me gusta tener contacto con mi bebé. un individuo, que forma parte del 3.Les hablo a los demás sobre mi bebé rol parental. La unión es uno de 4. Disfruto estar con mi bebé.

los conceptos que se pueden 5 . Sé que mi bebe necesita de mí.

interpretar como vínculo afectivo, 6. Siento felicidad cuando mi bebé está alegre al relacionar el concepto dado por 20. Me da gusto sentir a mi bebé.

Mercer. (Mercer, 1986).

Factor tres: Concepto Apoyo Informativo

Ayuda al individuo (madre-padre) nuevas.

a solucionar problemas por sí solo 11. Disfruto tener a mi bebé cerca de mí.

ofreciéndole información útil para 12. Siento que soy importante para mi bebé

cada problema y/o situación. 13. El tiempo que paso con mi bebé es especial.

(Mercer, 1986).

21. Estoy orgulloso (a) de mi bebé.

23. Estoy pendiente de mi bebé mientras duerme.

Factor Cuatro: concepto Estrés

Sucesos de la vida percibidos de 9. Estoy contento(a) de que este bebé sea mío.

modo positivo y negativo y 17 . Pienso en mi bebé.

variables ambientales. (Mercer, 18. Reconozco las expresiones y gestos de mi 1986). bebé.

22. Estoy pendiente de los cuidados de mi bebé.

Fuente. Vargas-Vásquez, A. (2016). Validación del Instrumento "Vínculo Entre Padres E Hijos Neonatos" a partir del Maternal Attachment Inventory. Tesis requisito para optar al título de: Magíster en Enfermería Universidad Nacional de Colombia. Pág.137 - 138. Disponible en: http://bdigital.unal.edu.co/52842/1/52713456.2016.pdf 


\section{DISCUSIÓN}

Contar con instrumentos válidos y contextualizados facilita a los profesionales de la salud y en este caso a enfermería detectar factores protectores y de riesgo en una situación de cambio para la familia como lo es el nacimiento de un hijo.

La importancia que tiene la consolidación del vínculo afectivo entre madres, padres e hijos durante los primeros días de vida, despierta especial interés para enfermería, ya que el nacimiento de un hijo requiere la reorganización de roles y el afrontamiento de sentimientos a nivel personal y familiar por parte de los padres; sin dejar de lado las diferencias que este comportamiento tiene en cuanto al género, la cultura, la edad, las condiciones psicosociales del entorno, condiciones de salud de los hijos, el medio hospitalario, entre otras razones, tal como lo afirman diversos expertos (19-21).

El vínculo afectivo entre padres e hijos es constantemente referido por investigaciones de enfermería y de otras disciplinas, en las que se resalta la importancia de fomentarlo para contribuir al futuro del país $(22,23)$.

Esta cercanía proporciona al bebé seguridad y gratificación, y sirve de patrón o representación interna para todas las relaciones de apego posteriores, por su parte el adulto también manifiesta determinados comportamientos como respuesta a las expresiones de apego del bebé. Estos comportamientos recíprocos llevan al desarrollo de una relación compleja y duradera, el apego ${ }^{(24) .}$

El establecimiento del vínculo afectivo entre las madres, los padres y su hijo nacido a término contribuye a la adecuada estructuración psíquica y cognitiva de los neonatos para su desarrollo y supervivencia durante todas las etapas del ciclo vital, generando a futuro seguridad, confianza, autoestima, madurez y responsabilidad como padre o madre $(19,25,26)$.

El cuidado de enfermería al neonato implica en sí mismo el cuidado de su familia, en especial de sus padres, pero para poder brindar cuidado familiar en torno al cuidado neonatal resulta necesario la valoración con indicadores empíricos válidos para la práctica, que faciliten a la enfermera en diferentes contextos como: las unidades de cuidado neonatal, los servicios de ginecobstetricia, la sala de partos, el alojamiento conjunto o en la visita al hogar en el posparto, el reconocimiento de las emociones, aspiraciones, necesidades de cuidado, fortalezas, condiciones y diversidades culturales de cada familia, para planificar el cuidado con base en las necesidades reales y especificas identificadas dentro de los procesos de interacción padres-hijo ${ }^{(27-}$ 29).

Al no contar con instrumentos válidos en el contexto colombiano para medir el vínculo afectivo entre padres e hijos neonatos nacidos a término, se realizó la validación transcultural del instrumento Maternal Attachment Inventory (MAI) con el debido rigor metodológico: Traducción, retro-traducción por expertos y traductores oficiales, asesoría lingüística y estadística en sus fases de validación facial, de contenido y de constructo, este ejercicio enriquecedor evidencia la necesidad de un adecuado proceso de adaptación semántica de instrumentos en cada contexto, y llevó a concluir a las investigadoras y la autora del MAl que el resultado fue un nuevo instrumento válido para Colombia pero diferente al original. 
Los resultados de la validez de constructo, aportan evidencia para inferir que el nuevo instrumento mide el fenómeno para el cual fue diseñado: vínculo afectivo entre padres e hijos neonatos definido como las expresiones y comportamientos verbales, gesticulares y de signos entre madres y/o padres y neonatos, que establecen la relación específica y privilegiada dada por la unión permanente que se inicia incluso desde la preconcepción y se consolida primordialmente en las primeras horas posteriores al nacimiento ${ }^{(6)}$.

La construcción de un instrumento para medir el vínculo a padres de neonatos nacidos a término que durante su diseño tiene en cuenta las posibles variables que describe la literatura como influyentes en la consolidación vincular, con el fin de obtener resultados con la menor cantidad de sesgos posibles, busca dar seguridad durante su aplicación; como ejemplo de situaciones que interfieren en el vínculo tenemos la edad gestacional al momento del parto, ya que la condición de prematurez ha presentado menor puntuación vincular al momento de medición del vínculo, otra situación que influye y debe tenerse en cuenta cuando se mide el vínculo afectivo es la condición de salud del recién nacido y la materna con referencia a la separación familiar que implica esta condición, pues cuando se ha medido la relación vincular en comparación de neonatos sanos y de neonatos hospitalizados, se describen como variables que afectan el vínculo la hospitalización, los procedimientos invasivos y la dificultad que tienen los padres para poder interactuar con sus hijos en el entorno de la unidad neonatal, el cual describen como hostil. (30).

\section{CONCLUSIONES}

El instrumento Vínculo afectivo entre padres e hijos neonatos es válido y confiable para ser utilizado en la práctica de enfermería tanto en las Unidades de Cuidado Neonatal como en el escenario ambulatorio para medir el vínculo de los diadas madre-hijo o padre-hijo.

La rotación Varimax como método estadístico de valoración de la estructura del constructo del instrumento agrupó los ítems de acuerdo con los valores coincidentes en cuatro factores, que en su contenido presentan relación con los conceptos de la teoría de Convertirse en madre de Ramona Mercer, ellos son: apoyo emocional, unión-interacción, apoyo informativo y estrés.

Para la disciplina de enfermería tener instrumentos empíricos válidos y confiables amparados en constructos teóricos propios contribuye a la formulación de propuestas de intervención susceptibles de medir, en este caso propuestas dirigidas al grupo materno perinatal para el fortalecimiento del vínculo afectivo entre padres e hijos que apunten al bienestar de los niños en su desarrollo.

Por ser el puerperio una etapa de intensos cambios en las diferentes dimensiones del ser humano, que involucran no solo a la madre en su ejercicio de la maternidad sino al recién nacido es importante para enfermería posibilitar momentos como el contacto piel a piel, el inicio temprano de la lactancia materna, el alojamiento conjunto madre hijo en los servicios de ginecoobstetricia, para así dar fuerza a las conductas de apego y favorecer el desarrollo futuro del infante.

Reviste importancia la utilización del instrumento producto de esta investigación reconociendo la autoría y ofreciendo la información completa de los resultados de 
este a los autores para continuar fortaleciendo las propiedades psicométricas del mismo,

\section{REFERENCIAS}

1. Bowlby, J. Una Base segura; apego y salud en el desarrollo humano. Barcelona: Paidós. 1989.

2. Ainsworth, M-D.; Bell, S-M. Apego, exploración y separación, ilustrados a través de la conducta de niños de un año en una situación extraña. Vol. 1. 1970.

3. Kennel, J. y Klaus, M. Apoyo pediátrico para los padres. En: Atención primaria en pediatría. $4^{\circ}$ edición. España: Océano. 2002. p. 619-627.

4. Mercer, R. The relationship of developmental variables to maternal behaivor. Rev. Nurs Healt, Vol. 9. 1986. p. 25-33.

5. Beal, J-A. Methodological issues in conducting research on parent-infant attachment. Rev. Journal of Pediatric Nursing, Vol. 6. 1991. P. 11-15.

6. Müller, M. Cuestionario para medir el apego materno-infantil. Rev. Nurs Meas. Vol. 2 n. 2. 1994. p. 129-141.

7. Yu, C.-Y., Hung, C.-H., Chan, T.-F., Yeh, C.-H. Lai, C.-Y. Prenatal predictors for father-infant attachment after childbirth. J Clin Nurs. Vol. 21. n. 11. 2012. p. 1577-1583.

8. Vargas, V. Validación del instrumento: "Vínculo entre padres e hijos neonatos" a partir del Maternal Attachment Inventory [tesis de maestría en Internet]. Bogotá: Universidad Nacional de Colombia, Facultad de Enfermería; 2016 [acceso: 19 may 2017]. Disponible en: http://www.bdigital.unal.edu.co/52842/1/52713456.2016.pdf

9. Polit y Hunter (2000). Investigación científica en ciencias de la salud. $6^{a}$ ed. Mc Graw Hill Interamericana.

10. Audi, Robert. The Cambridge Dictionary of Philosophy. 2 Edición. Ed. Cambridge University Press. EEUU. 1999.

11.Streiner, D.L. Starting at the beginning: an introduction to coefficient alpha and internal consistency. Rev. Pers Assess, Vol. 80. 2003. p. 99-103.

12. Beaton D.E., Bombardier C., Guillemin F. y Ferraz M.B. Guidelines for the process of cross-cultural adaptation of self-report measures. Rev. Spine. Vol. 25. n. 24. 2000. p. 3186-3191.

13. Tristán-López, A. Modificación al Modelo de Lawshe para el Dictamen Cuantitativo de la Validez de Contenido de un Instrumento Objetivo. Rev. Avances en Medición. 2008. Vol. 6, p. 37-48.

14. Fleiss, J. L. Measuring nominal scale agreement among many raters. Rev. Psychol Bull. Vol. 76. n. 5. 1971. p. 378-382. Recuperado de: http://psycnet.apa.org/index.cfm?fa=buy.optionToBuy\&id=1972-05083-001.

15. Pett, M., Lackey N., Sullivan J. Assessing the characteristics of matrices. Rev. Making Sense of Factor Analysis. Thousand Oaks Sage, 2006. p. 51-84.

16. Oviedo, H. C. y Campo Arias, A. Aproximación al uso del coeficiente alfa de Cronbach. Rev Col Psiqui, vol. 34. n. 4. 2005. p. 572-580.

17. Dalenius, T. Hodges, J. Minimun variance stratification. Rev. Journal of the American Statistical Association. 1959. Vol 55. n. 285. p. 88 -101.

18. Guisan De González, C. Vaamonde A. y Barreiro A. Análisis multivariante: Métodos factoriales. En: Tratamiento de datos con $R$, Statistica. $1^{\circ}$ edición. España: Ediciones Díaz de Santos.2011. p. 677-703. 
19. Trupe, R-D.; Macfie, J.; Skadberg, R-M.; Kurdziel, G. Patterns of emotional availability between mothers and young children. Rev. Associations with risk factors for borderline personality disorder. . [Online]. Vol 27. n. 12017 [Citado 18/01/2019]. Disponible en: $\quad$ https://doi.org/10.1002/icd.2046. https://onlinelibrary.wiley.com/doi/epdf/10.1002/icd.2046

20. Mite Cárdenas, GV. Vínculo afectivo madres - hijos neonatos a término según edad y paridad: una comparación. Universidad nacional de Colombia, Facultad de Enfermería. [Online]. 2017. Tesis para optar por el título de magister en Enfermería. 2017. Disponible en: http://bdigital.unal.edu.co/61176/1/Tesis\%20\%2010\%20DICIEMBRE\%20201 7\%20\%282\%29.pdf

21. Muñoz-Molina, M., Poo-Figueroa, A-M., Bustos-Medina, L., BaezaWeinmann, B. Acuerdo entre tres evaluadores y un experto en la detección de riesgo relacional en el posparto entre la madre y su recién nacido, Temuco, IX región, Chile, 2010. Rev. Colombiana de Obstetricia y Ginecología. [Online]. Vol. 65. n. 2. 2014. p. 129-138. [Citado: 2019/01/22]. Disponible en: <https://revista.fecolsog.org/index.php/rcog/article/view/61/61

22. Bornstein $\mathrm{MH}$, Suwalsky JT, Breakstone DA. Relaciones emocionales entre madres y bebés: conocimientos, incógnitas e incógnitas desconocidas. Rev. Desarrollo y psicopatología. [Online]. Vol. 24. n. 1. 2012. p. 113-23. Disponible en https://www.ncbi.nlm.nih.gov/pmc/articles/PMC3426791/

23. Vega, F. L. Importancia de fomentar el vínculo de apego en la infancia. Rev. Mex Pediatr, Vol. 77. n. 3. 2010. p. 103-104.

24. Rodríguez-Holguín, Y.A. y Muñoz De Rodríguez, L. Las madres se adaptan a la hospitalización de sus hijos mediante la exploración y el apoyo. Rev. Actual Enferm, Vol. 12. n. 1. 2009. p. 12-18.

25. Farkas, C., Santelices, M., Aracena, M. y Pinedo, J. Apego y ajuste Socio Emocional: Un Estudio en Embarazadas Primigestas. Rev. Psykhe. Vol. 17. n. 1. 2008. p. 65-80.

26. Restrepo-Montoya, D. Influencia del vínculo afectivo madre/hijo en el desarrollo de la autonomía de los niños en situación de discapacidad, Fundación Ángeles de amor, Ibagué Tolima. Trabajo final para optar por título de Psicólogo. Universidad Nacional Abierta y a Distancia. Escuela de Ciencias Sociales, Artes y Humanidades. [Online]. UNAD-CEAD, Ibagué. 2013. [Citado 18/01/2019]. Disponible en: https://repository.unad.edu.co/bistream/10596/2286/1/110505939.pdf

27. Suaréz- Delucchi, N. \& Herrera, P. La relación del hombre con su primer(a) hijo(a) durante los primeros seis meses de vida: Experiencia vincular del padre. Rev. Psykhe. [Online]. Vol. 19. n. 2. 2010. p. 91-104. [Citado 18/01/2019].

Disponible

en: http://dx.doi.org/10.4067/s0718/22282010000200009

28. Muñoz, M.; Poo, A-M.; Baeza, B.; Bustos, M-L. Riesgo relacional madrerecién nacido: Estudio de prevalencia y variables asociadas. Rev. chil. pediatr. [Internet]. Vol. 86. n. 1. 2015. p. 25-31. [Citado 18/01/2019]. Disponible https://scielo.conicyt.cl/scielo.php?script=sci_arttext\&pid=S037041062015000100005\&Ing=es.

29. Barbosa-Merighi, M-A.; Pinto de Jesus, M-C., Ribeiro-Santin, K., Moura de Oliveira, D. Cuidar del recién nacido en la presencia de sus padres: vivencia de enfermeras en unidad de cuidado intensivo neonatal. Rev. Latino-Am. Enfermagem. [Internet]. Vol. 19. n. 6. 2011. Disponible en: http://rlae.eerp.usp.br/ 
30. Alonso Allende, L., González Fuente, L. J., Pérez Rivera, F. J., \& Fernández García, D. Apego en el postparto precoz: comparación entre madres de neonatos ingresados en el Servicio de Obstetricia y en la Unidad de Cuidados Intensivos Neonatales. Rev. Enfermería Global. Vol. 16. n. 45. 2017. p. 295-308. 